\title{
Adaptation of Candida parapsilosis to hospitalized infant microbiomes
}

Patrick T. West

Samantha L. Peters

Matthew R. Olm

Feiqiao B. Yu

Haley Gause

Yue Clare Lou

Brian A. Firek

Robyn Baker

Alexander D. Johnson

Michael J. Morowitz

Robert L. Hettich

Jillian Banfield

\section{Video Byte}

Keywords: microbial eukaryotes, Candida, metagenomics, in situ, genome-resolved metagenomics, straintracking, hospital microbiome, neonatal intensive care unit, premature infants, Microbiome

Posted Date: October 13th, 2021

DOI: https://doi.org/10.21203/rs.3.rs-967284/v1

License: (9) This work is licensed under a Creative Commons Attribution 4.0 International License.

Read Full License 


\section{Abstract}

Cases of the fungal disease candidiasis in infants have been increasing over the past two decades, but our understanding of the behavior and evolution of Candida spp. outside laboratory models has been lacking. Researchers assembled new C. albicans and C. parapsilosis genomes using data from premature infant feces, hospital surfaces, clinical samples, and non-hospital environmental samples. Relative to an existing reference genome, the $\mathrm{C}$. parapsilosis genomes analyzed had genomic "hotspots" of single nucleotide variants, and the $\mathrm{C}$. parapsilosis genomes from premature infants had hotspots that matched hospital and subway surface samples, which may suggest a common ancestor. The researchers also analyzed a microbial gene expression time course in one infant being treated for Candida infection. The in situ transcriptomic profiles were highly variable relative to cultured C. parapsilosis and genes associated with biofilm formation tended to have lower expression in situ relative to cultured samples of the same strain. Although the gene expression data presented here is only from one infant, these results underscore the importance of in situ research, and the genomic results outline potential evolutionary processes and hospital strain persistence. 